\title{
RETROSPECTIVE ANALYSIS OF INCIDENCE OF POSTOPERATIVE COMPLICATIONS IN BARIATRIC SURGERY IN THE GENERAL HOSPITAL OF VALENCIA
}

Hernández M.J. ${ }^{1}$, Torres O. ${ }^{2}$, Lorena G. ${ }^{1}$, Bruna M. ${ }^{2}$, Hernández J. ${ }^{1}$, de Andres J.

${ }^{1}$ Hospital General Universitario de Valencia, Dept of Anaesthesiology \& Intensive Care, Valencia, Spain,

${ }^{2}$ Hospital General Universitario de Valencia, Dept of Surgery, Valencia, Spain

\section{Background}

Obesity is a global health problem. Up to $35 \%$ of the population in the US and $15-20 \%$ in Europe are considered obese. Obesity is associated with significant comorbidities. In recent years there has been an increase in the number of bariatric surgeries. Approximately 344,000 bariatric surgeries are performed annually worldwide. Pre-anesthetic assessment should be focused on detection of co-morbidities and high-risk patients to optimize outcome.

\section{Objetive}

To determine the incidence of mortality in our study population and the most frequent complications that have occurred in patients undergoing bariatric surgery from November 2010 to November 2015 in the General Hospital of Valencia.

\section{Materials and Methods}

A retrospective observational analysis of bariatric surgeries performed in our hospital between November 2010 and November 2015.

\section{Results}

Between November 2010 and November 2015, 141 bariatric surgery procedures were performed in our hospital. The specific interventions include 123 sleeve gastric, 15 gastric bypass, and only 3 intragastric prosthesis. The main comorbidities found were, obesity sleep apnea (75 patients), hypertension (76 patients), metabolic syndrome (66 patients), dyslipidemia (60 patients), type 2 diabetes mellitus (60 patients), joint disorder (47 patients) and heart disease (6 patients). The 30-day mortality rate was $0 \%$. 8 patients presented surgical wound abscess, 7 patients had respiratory infection without requiring critical care unit admission, in 7 patients suture dehiscence occurred and 8 patients had a intraabdominal hematoma, 1 patient suffered a perioperative myocardial infarction and reintervention were performed in 10 patients.

\begin{tabular}{lcccc}
\multicolumn{5}{c}{ Postoperatory morbimortality } \\
& $\begin{array}{l}\text { Global } \\
\mathbf{n}(\%)\end{array}$ & $\mathbf{2 0 1 0 - 1 2}$ & $\mathbf{2 0 1 3 - 1 5}$ & $\mathbf{n}$ \\
\hline & 0 & 0 & 0 & \\
Mortality & $28(19)$ & $15(19,0)$ & $13(21,0)$ & $\mathrm{ns}$ \\
Morbidity & $8(5,7)$ & $4(5,1)$ & $4(6,5)$ & $\mathrm{ns}$ \\
Wound infection & $7(5,0)$ & $5(6,3)$ & $2(3,2)$ & $\mathrm{ns}$ \\
Respiratory complications & $7(5,0)$ & $5(6,3)$ & $2(3,2)$ & $\mathrm{ns}$ \\
Gastric leak & $3(2,1)$ & $2(2,5)$ & $1(1,6)$ & $\mathrm{ns}$ \\
Plasty stenosis & $7(5,0)$ & $3(3,8)$ & $5(8,1)$ & $\mathrm{ns}$ \\
Hemoperitoneum & $1(0,7$ & 0 & $1(1,6)$ & $\mathrm{ns}$ \\
\hline Biliar leak & $1(0,7$ & $1(1,3)$ & 0 & $\mathrm{~ns}$ \\
AMI & $1(0,7)$ & 0 & $1(1,6)$ & $\mathrm{ns}$ \\
Upper digestive bleeding & $10(7,1)$ & $7(8,9)$ & $3(4,8)$ & $\mathrm{ns}$ \\
Reinterventions & & & &
\end{tabular}

1 year comorbidity evolution

$\begin{array}{ccccc} & \mathbf{N}^{\circ} & \begin{array}{c}\text { Improve } \\ \mathbf{n}(\%)\end{array} & \begin{array}{c}\text { Cure } \\ \mathbf{n}(\%)\end{array} & \begin{array}{c}\text { Fails } \\ \mathbf{n}(\%)\end{array} \\ \begin{array}{c}\text { HBP } \\ \text { Dyslipemia }\end{array} & 53 & 11(20,8) & 39(73,6) & 3(5,7) \\ \text { Type 2 DM } & 43 & 9(17,8) & 35(77,8) & 2(4,4) \\ \text { OSA } & 46 & 11(23,9) & 25(58,1) & 9(20,9) \\ \text { Arthrosis } & 28 & 11(39.3) & 10(35,7) & 7(25) \\ \end{array}$

\begin{tabular}{cccc}
\multicolumn{4}{c}{ Weight loss } \\
\hline Months & Cases & Weight lost (Kg) & $\%$ BMl excess \\
\hline 3 & 99 & $28,4+/-11,2(1-61)$ & $44,3+/-14,7(2-78)$ \\
\hline 6 & 95 & $36,4+/-14,2(-2-77)$ & $57+/-17,5(-5-97)$ \\
\hline 12 & 95 & $45,1+/-16,3(5-92)$ & $69,5+/-19,4(16-107)$ \\
\hline 24 & 53 & $45+/-17,5(8-93)$ & $69,2+/-22,2(13-102)$ \\
\hline 36 & 17 & $35,9+/-17(10-72)$ & $61+/-23(25-93)$ \\
\hline 48 & 9 & $30,2+/-15,3(11-50)$ & $56,3+/-29,1(24-101)$ \\
\hline 60 & 3 & $30,2+/-5,2(34-43)$ & $73,6+/-31,6(31-101)$ \\
\hline
\end{tabular}

\section{Conclusions}

The absence of deaths associated with bariatric surgery and the low incidence of complications associated with it, make bariatric surgery a safe technique for the patient, which provides great benefits on health. Anesthetic management involves challenging and extensive knowledge of the physiology and pharmacology variations in the obese patients. 